\title{
Chefs Know More than Just Recipes: Professional Vision in a Citizen Science
}

Game

Marisa Ponti, Igor Stankovic, Wolmet Barendregt, Department of Applied

Information Technology, University of Gothenburg,

Bruno Kestemont, Lyn Bain (Foldit Players, Go Science Team).

\section{Introduction}

Over the past decade there has been a rapid increase in the number of citizen science projects. Citizen science is now an accepted term for a range of practices that involve members of the general public, many of which are are not trained as scientists, in collecting, categorizing, transcribing or analyzing scientific data (Bonney et al., 2014). Successful citizen science projects include involving participants in classifying astronomical images, reporting bird sightings, counting invasive species in the field, using spatial reasoning skills to align genomes or folding proteins. These projects feature tasks that can only be performed by people (e.g., making an observation) or can be performed more accurately by people (e.g., classifying an image) with the support of computational means to organize these efforts. As such, citizen science often relies on some form of socio-computational system (Prestopnik \& Crowston, 2012).

Games are one form of such socio-computational systems used in citizen science to entice and sustain participation. The list of citizen science games that people can play while contributing to science is growing. Games, and gamified websites or applications, take a range of forms within citizen science. Some projects, like MalariaSpot, include just a few game elements such as points, badges, and leaderboards. Other projects, like Foldit and Eyewire, are full immersive experiences. Still others, such as Forgotten Island, are beginning to use narrativebased gamification approaches. Depending on the complexity of task they feature, citizen science games can be classified in microtasks and megatasks (Good \& Su, 2013). Microtasks can be accomplished quickly and easily by anyone who can read some simple instructions, while megatasks concern challenging problems whose solution might take weeks or even months of effort from qualified experts. The goal of involving the general public to accomplish megatasks is to have a 
large pool of candidates from which to identify few talented individuals whose skills can solve challenging problems. Good and Su identified hard games as an approach to megatasks, which give players access to small numbers of extremely challenging individual problems. A very successful example of hard game is Foldit which invites talented players to predict three-dimensional (3D) models of proteins from their amino acid composition. Scientists analyse players' highest scoring solutions and determine whether or not there is a native structural configuration that can be applied to relevant proteins in the real world to help eradicate diseases and create biological innovations.

As skilled and talented as they can be, Foldit players would struggle to solve those complex puzzles without the support of machines. Foldit is a good example of human computation (von Ahn, 2005), since it was developed to find places where computational power is most useful and where human abilities are best applied (Cooper, 2014). Soon after the release of Foldit, players themselves strengthened human computation by requesting the addition of automation in the form of recipes, small scripts of computer code that automate some protein folding processes, to carry out their strategies more easily when solving puzzles. Over the years, the increasing use of recipes has created resentment in several players who think that recipes have taken too much place in the game and make novices think that running recipes is all they need to play the game ${ }^{1}$. However, previous findings (Ponti \& Stankovic, 2015) suggest that the use of recipes allows skilled Foldit players to strengthen their role of experts rather than becoming appendices of automated gameplay. Skills are part of players' "professional vision" (Goodwin, 1994). The main purpose of this study is to investigate players' professional vision and interpret their use of recipes during their gameplay. The main research question is: What do players observe and do when they use recipes in their gameplay? To address this question, we examined the choices made by players solving two different kinds of puzzles, a beginner's puzzle and an advanced one. Specifically, we studied when, how and why the players ran recipes when solving the puzzles, and what actions those recipes performed in the gameplay. 
The overall goal is to contribute to the knowledge of the relationship between technology and skills in performing megatasks and to draw implications for the design of citizen science games based on human computation.

\section{Related Work}

To place this study in context, we thus present an overview of the debate on the relationship between technology and skill. We reviewed the literature selectively to identify ways to interpret this relationship and examine embedded assumptions and theoretical problems.

\section{The Relationship between Technology and Skills}

"Skill" is an elusive concept, as it is defined in several ways. Carmeli and Tishler (2006) define skill loosely as the "ability to do something in an effective manner' (p. 13). In the context of digital games, Che Pee (2011, p. 22) defines competence as a "combination of knowledge, skills, and behavior leading to an individual being able to perform a certain task to a given level.' Schell (2008) classified the skills that are required from players to play a game into physical, mental and social skills. A concern with defining and classifying skills is the attempt to decontextualize them and see them in static isolation from specific tasks to be accomplished at specific times by different groups of people.

Decontextualizing skills also implies neglecting the environment in which people interact with materials and tools, as opposed to simply using them for a task. Reeves, Brown, and Laurier (2009) also noted that several studies of games tended to neglect the ecology of social settings in which expertise is produced and recognized, and focused on measuring experts and their expertise.

More recently, Tedder (2016) argued that skilled relationships with tools including technologies - need ecological consideration, as they need to be understood in their enactment, instead of "decomposing" skills, tasks, and technologies into entities that do not mutually influence each other.

An example of ecological consideration is Linderoth's (2010) study of the relationship between games and learning. Using Gibson's ecological approach, Linderoth (2010) argued that progressing in a game, being able to take actions and reach built-in game goals, are not solely a matter of learning. Since games 
can show affordances, for example by highlighting the available objects to interact with, a player not always needs to learn to differentiate between the available information in the gaming domain. Linderoth concluded that gaming may demand less learning or skills by players than, for example, reading sheet music.

In other accounts of skilled relationships with technologies, such as those provided by labor process studies, the role of technology is not overlooked but seems to be misplaced. Several labor process studies, starting with Bravermann (1974), posit a direct relationship between the use of technology and deskilling, suggesting that human skills are replaced by being incorporated in technologies. A link between the deskilling thesis and games was made by De Paoli (2013), who examined the use of bots in Massively Multiplayer Online Role Playing Games (MMORPGs), and argued that this type of automatic play can reduce proficiency of players, since their skills would get incorporated into the bots. A major criticism levelled at the deskilling thesis stems from ethnomethodologists, who point to the lack of empirical evidence supporting the simplistic association of technological development and skills and the assumption that a given set of skills is necessary to accomplish certain tasks (Rouncefield, Slack, \& Hartswood, 2011). These critics argue that the essentialist view of skills neglects that many tasks are multiskilled and that skills mesh together. In their overview of relevant sociological approaches to study the relationship between skills and technology, the same critics also examined the approach to skill as a form of knowledge often informal, tacit and the product of the acquisition of the local culture in which a specific task is accomplished. In this view, skill is a competence necessary for mutual intelligibility on the part of the members of a group. It includes, for example, "knowing where others are in their work" and "getting round the inadequacies of the system." (Rouncefield, Slack, \& Hartswood, 2011, p. 207). Hardly is this competence incorporated in a decontextualized notion of skill or embedded in software. In this paper, we do not embrace a notion of skill as an abstract and decontextualized entity, but we see it as an ability situated in the gameplay, which 
players develop by engaging actively with objects and tools within the game and the local community surrounding the game. Taking this approach means that we do not conceptualize skills as "stand-alone" cognitive processes or conceptual structures, but as abilities and forms of knowledge being performed through a network of connections-in-action involving humans and technologies. For this reason, we turn to Goodwin's notion of professional vision.

\section{Professional Vision}

Theoretically, our work builds upon Goodwin's (1994) notion of professional vision, which he coined to refer to the specialized way in which members of a professional group look at a phenomenon of interest. Therefore, an archaeologist, a farmer or a builder see different things when looking at the same patch of dirt, because of their different professional visions. Goodwin explained that professions initiate their members into specialized ways of seeing and understanding phenomena in their area of interest. To do so, they use three practices: (1) coding, the use of language to describe and construct the object of investigation; (2) highlighting, which gives salience to specific phenomena in complex setting by marking them in some ways, and (3) producing material representations, which embed and structure the knowledge produced and make it available through time and space.

Thus, professional vision brings together and organizes:

- Gaze: ways of attending to and perceiving a phenomenon.

- Discourse: ways of talking about the observed phenomenon.

- Thinking: ways of interpreting the observed phenomenon.

- Producing artifacts: representing the knowledge produced.

This notion is relevant for our study because the ability to see and identify significant aspects is not an individual cognitive process, but a situated activity distributed between humans and non-humans, in which the interaction between the two becomes the object of investigation (Gherardi, 2012). In Foldit, we can say that players' professional vision involves the ability to see and interpret significant interaction with objects and tools in the game. 


\section{Foldit}

Foldit is a serious game developed by the Center for Game Science at the University of Washington in $2008^{2}$. Figure 1 shows a screenshot of the game.

Two years after launching, the game counted already 57,000 players (Lafourcade, Joubert, \& Le Brun, 2015). The main goals of Foldit include protein structure prediction and design, coevolution of human-computer relationship and automation of folding strategies from human players. Foldit is a challenging game whose mechanics include a complex of rules, strategies and moves associated with the prediction and design of a protein. For the sake of brevity and clarity, also considering the technical jargon which includes a mix of biochemistry and bioinformatics terms used to describe the functioning of the game, we refer the interested readers to sources like Wikia Foldit, the wiki created and maintained by players in order to understand the jargon.

The game has no story, setting, characters, or narratives. Instead, players must solve puzzle challenges to achieve the highest possible scores with a set of given tools. Players manipulate the structure of a protein in a 3D space to reach as high a score as possible. Players can play individually (soloist play) or as members of a team (evolver play). No knowledge of the scientific field the game is based on is required, but players but are expected to have several skills, especially excellent spatial awareness, the ability to take short-term risks for long-term gain, and the converse, recognizing a dead-end early and knowing when to quit (Hand, 2010).
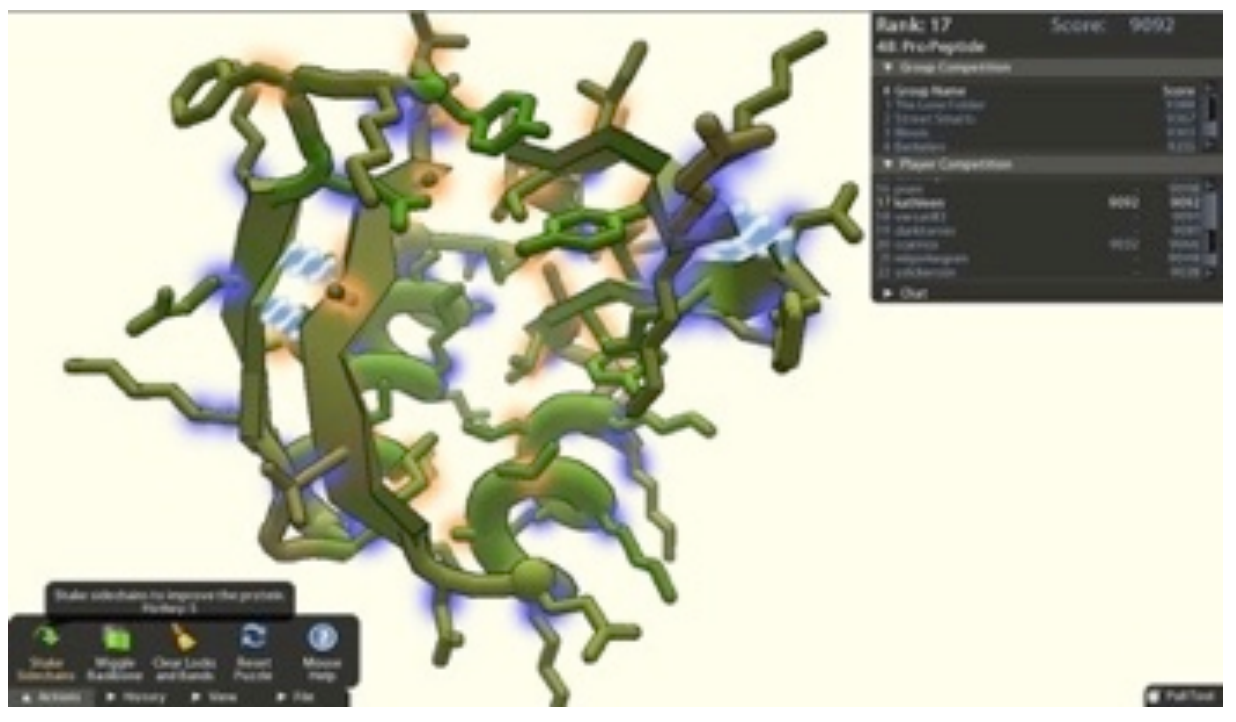
Figure 1. A screenshot of Foldit (Source: Fold.it)

\section{Foldit Recipes}

The Foldit development team gave players the opportunity to use and write and adapt recipes to manage the increasing complexity of the game (Cooper et al., 2011). Recipes are computer programs that allow players to interact automatically with a protein and repeat simple routines consistently, or perform a series of complex routines which keep running in the background for ever. The term recipes includes both GUI and Lua scripts. GUI recipes perform predefined actions such as adding or adjusting the strength of rubber bands (soft constraints which connect amino acids and pull on them independently of the player), restoring previous structures, and invoking optimization methods such as shake and wiggle (they both move the protein around a little to improve the score). Lua script recipes are based on a simple programming language called Lua, which players can use to create and modify recipes. Lua script recipes are more powerful than GUI recipes. They allow performing many more moves to solve complex puzzles involving more protein elements (segments, molecules, etc.) to work on. A subsequent more advanced version of Lua recipes also allows selecting options related to the stage of the gameplay in a puzzle (beginning, midgame, endgame). For example, it can help optimize a protein structure, either when starting a given structure, or when players get stuck with other recipes, or at the very end of the gameplay. Players can systematize these strategies using the Cookbook (Figure 2), a feature developed to help them write, share and run their recipes. Since the start of Foldit, players have created a sheer number of recipes (Khatib, et al., 2011). A number of recipes are publicly shared. Many others are private or shared only within a team. Using recipes is one of the easiest way to get to the top and, reportedly, most top players are using them to some extent (Ponti \& Stankovic, 2015). 


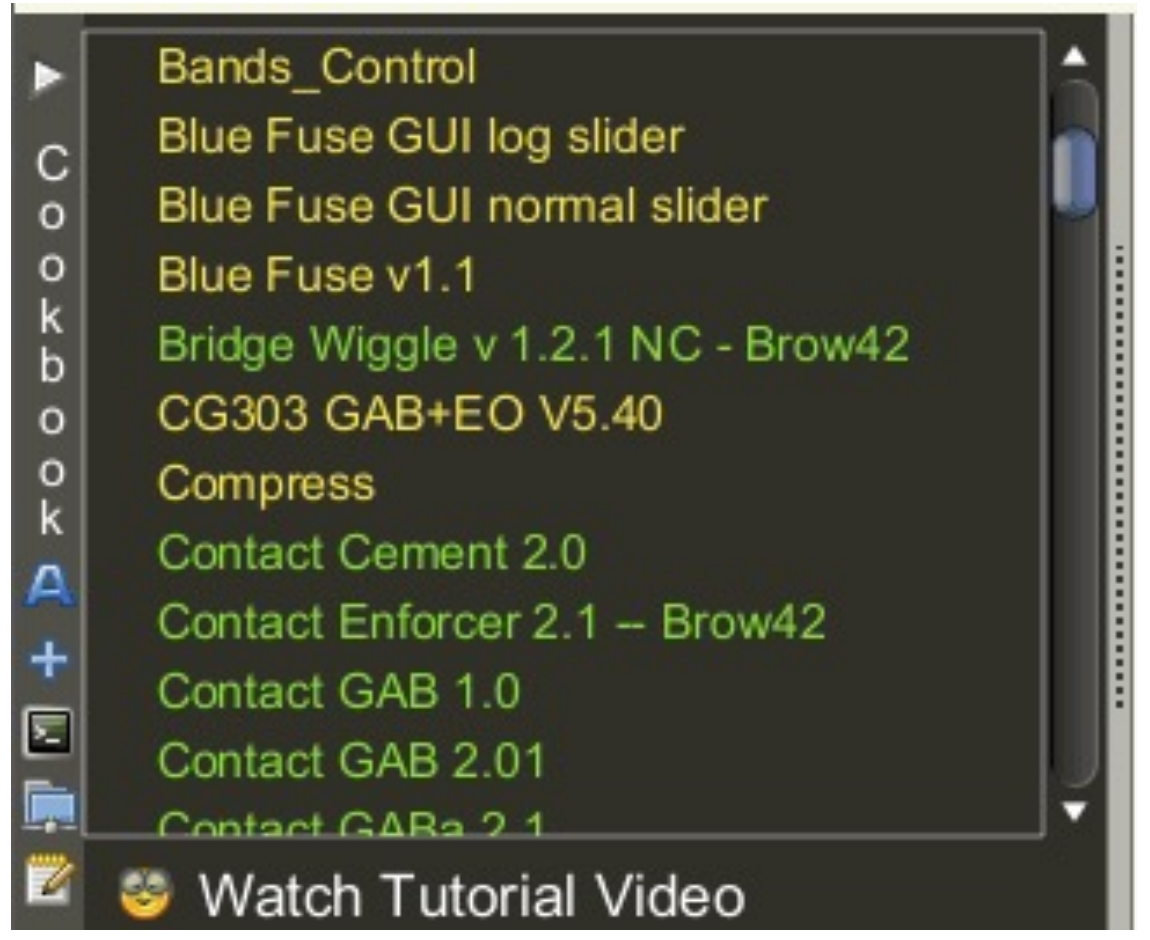

Figure 2. A screenshot of a cookbook containing different types of recipes (Source: Bruno Kestemont)

\section{Methods}

\section{Issues in the Study of Players' Professional Vision}

Players' professional vision allows observing and assessing the structure of the protein, understanding when a dead end is found and when they can take some risks in the short term for long-term advantage. The study of players' professional vision posed some practical challenges. These practical challenges influenced our methodological choices and the research question. Unfortunately, as rightly noted by Papeete, Janssen and Ostrom (2010), methodological discussions rarely acknowledge practical considerations and offer little guidance on how to address these limitations. We had to balance methodological demands under the conditions of limited access to data. First, because of competitive reasons we were not allowed logging in and accessing real-time data from gameplay. Second, gaining access to and recruiting potential research participants was also challenging because they were hard-to-reach. Several potential participants (contacted initially through the built-in mail in Foldit) did not respond to recruitment efforts either because of a general lack of interest, or because they no 
longer played the game. Over a year of exploring the game, through reading online discussions in the game forum and documents in Wikia Foldit, and interviewing a small group of players in a previous study (Ponti \& Stankovic, 2015), we managed to establish rapport with a top-ranked player. This expert player has invested a lot of time on playing the game, has handfolded for at least two years and has moved beyond simple competence to achieve high positions in the game ranking leagues. Third, playing Foldit involves a steep learning curve. It takes a lot of effort and time. Although one of the authors, Stankovic, played the game at a beginner's level to acquire a basic understanding of the gameplay (about 50 hours of play), it was soon clear it would take him an inordinate amount of hours to function as intermediate player. Since we were interested in studying professional vision at different levels of expertise, we kept Stankovic as a beginner and we relied on the top player to help us gain access to an intermediate player who is member of his team. This player has also played for two years, investing much time and computer power to be not too far behind the top players. The three players (beginner, intermediate and expert) were a purposive sample which allowed focusing on their respective abilities when playing the game. We considered ability as a player the characteristic that would best enable us to answer our research question.

\section{Data Collection and Analysis}

We asked the players to conduct autoethnography (Brown, 2015) on their playing of the puzzles. All three players played one beginner puzzle. Furthermore, we asked the expert player to keep a detailed record of his gameplay of one advanced puzzle. Both puzzles were De-novo puzzles in which only the primary sequence (series of amino acids) of a protein is known at the start. The peptide (chain) is provided as a straight string which players need to analyze and fold. The goal is to get close to a native low energy folded protein. Many strategies are provided by players to start this type of puzzles in tutorial pages and videos.

We asked the players to record their gameplay, if possible, and write detailed, first-hand accounts of their play. We chose this approach to be able to examine three accounts at different levels of expertise and knowledge of the game. 
Our unit of analysis was the observable interaction between players and their game space in the puzzles. Observation was informed by the concept of professional vision, as we looked at what players observed, did and thought (when thinking was made explicit and therefore became "observable") at different stages of the game and how they performed their actions, the actions performed by recipes and the outcomes of these actions. We also looked at the external sources of information used by the players. By external sources (as opposed to internal cognitive resources such as experience and intelligence) we refer to all the visual tools used by Foldit to convey information to players. The game interface displays text and numbers and contains many buttons and commands to interact with the game (see Figure 1).

The autoethnographic descriptions, including text, videos and screenshots, provided the opportunity to "see" how players described their playing and understood the unfolding of events in the game space. From these accounts we could infer their ability to see and interpret significant interactions with objects and tools in the game. The data corpus used in this study consists of four autoethnographic descriptions as is summarized in Figure 3.

\begin{tabular}{|c|c|c|}
\hline Player & $\begin{array}{l}\text { Beginner Puzzle }(<150) \text { : } \\
\text { Easy Mini Freestyle }{ }^{2}\end{array}$ & $\begin{array}{l}\text { Advanced: Unsolved De-Novo } \\
\text { FreeStyle } \mathbf{5 8}\end{array}$ \\
\hline $\begin{array}{l}\text { Igor (IS) - } \\
\text { beginner }\end{array}$ & $\begin{array}{l}\text { Video recording and text } \\
\text { description: } 6: 06 \text { minutes } \\
\text { before abandoning the game }\end{array}$ & \\
\hline $\begin{array}{l}\text { Lyn (LB) - } \\
\text { intermediate }\end{array}$ & $\begin{array}{l}\text { Text description of puzzle } \\
\text { completed in } 24 \text { hours, } \\
\text { including overnight recipes } \\
\text { when she was off. }\end{array}$ & \\
\hline $\begin{array}{l}\text { Bruno (BK) } \\
\text { - expert }\end{array}$ & $\begin{array}{l}\text { Video recording when he } \\
\text { was present and paused } \\
\text { when running recipes and he } \\
\text { was off: total } 16 \text { minutes of } \\
\text { man work and about } 3-4 \\
\text { days of computer work. }\end{array}$ & $\begin{array}{l}498 \text { minutes of man work (= about } \\
8 \text { hours) plus } 60 \text { minutes of editing } \\
\text { a recipe. } \\
6,5 \text { days of computer work on a } \\
\text { mean of about } 3 \text { tracks = about } 468 \\
\text { hours of computer time. } \\
\text { The final result was achieved with } \\
\text { about } 2 \% \text { of human resources and } \\
98 \% \text { of computer (recipes) work. }\end{array}$ \\
\hline
\end{tabular}

Figure 3. Summary of collected data 
We analyzed all the data collected using a grounded theory approach (Charmaz, 2006). Following an open approach to coding, we built codes as we analysed the data. In the second stage of axial coding, to address our central question "What do players observe and do when they use recipes in their gameplay?", we focused the analysis on the code "running a recipe" to provide a detailed account of player's actions that were conducive to decide to run a recipe. We used Scott's (2004) guiding questions for constant comparison of all the incidents coded with "running a recipe" across the four autoethnographic accounts and for examining the relationships between "running a recipe" and other categories identified through open coding. To identify main themes, we measured the frequency of occurrences of categories of actions related to "running a recipe" in each account and then across the four accounts. To ensure the rigor of the method, we used the member checking technique to verify the accuracy of the analysts' interpretation. For example, the process of axial coding and comparison within individual accounts was checked by each player to ensure the results of the analysis were credible and consistent to them.

\section{Results}

\section{Findings: Beginner Puzzle (<150): Easy Mini Freestyle}

In the three accounts, the players described different strategies to solve the same puzzle and, accordingly, their selection criteria for running recipes varied. Figure 4 summarizes the results of the axial coding analysis related to the most frequent actions performed by players in association to "running a recipe." 


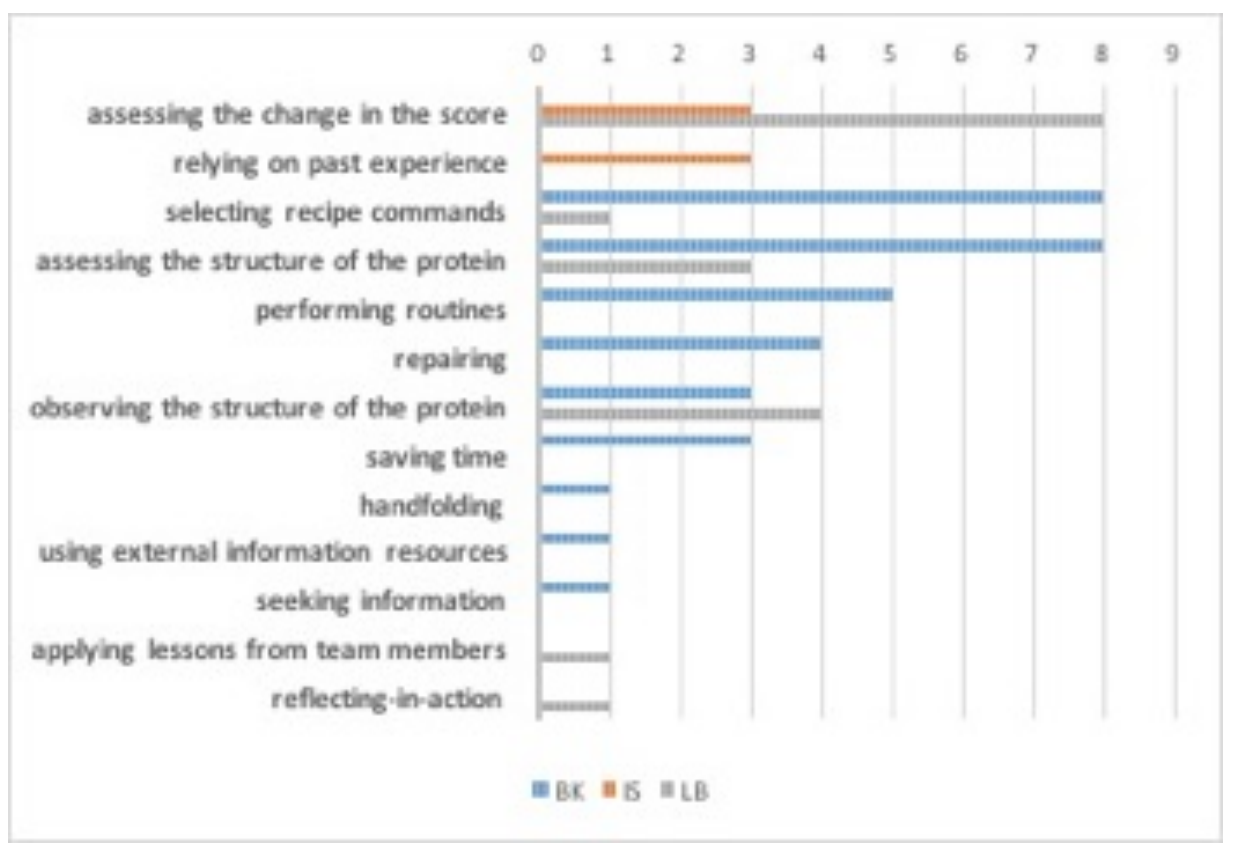

Figure 4. Actions associated to run a recipe in the beginner game

After counting the occurrences of categories of observed actions associated with "running a recipe" in each account, we found that 9 main actions were associated to running a recipe in BK's account, 7 main actions were associated in LB's account, and 2 in IS's account. The chart indicates that assessing and observing the structure of the protein are central categories in the gameplay of the expert player. The difference between the two can be articulated as follows: observation provides a picture of what the player sees, while assessment is an evaluation of what has been observed, including ideas of what could be done to improve the structure protein. The chart indicates that the two less experienced players are more concerned with assessing the output of their actions, including running recipes, on the score.

Figure 5 shows the occurrences of external sources of information used by the players to when performing the actions shown in Figure 4. 


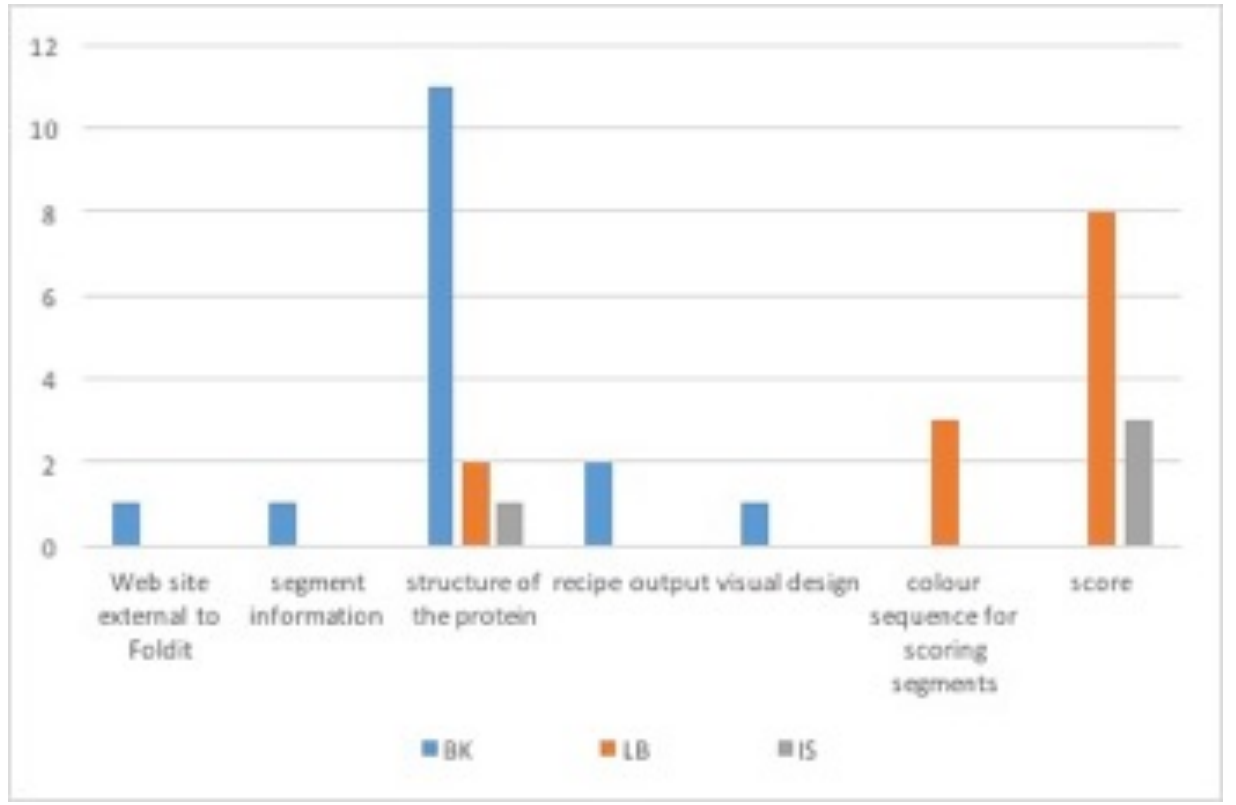

Figure 5. Most frequently used external sources of information

The axial coding helped us develop a major theme which we called "seeing and interacting with material representations." Below, we provide the accounts of what the three players observed, did and thought at different stages of the game, along with information on how they performed their actions. These narratives exemplify how the players expressed their professional vision (or lack of) through their practices of coding, highlighting and producing material representations, to help us understand what they did.

\section{Seeing and Interacting with Material Representations}

\section{Beginner: Assessing the Change in the Score}

IS started by observing the structure of the protein and rotating it to see the length of the protein. Then he did some hand-folding using a mix of random actions and knowledge of the most basic Foldit tools, such as wiggle and shake, which he had developed by playing the tutorials. However, given his little experience with the game, he felt unsure of the output of his actions. At a given point, he seemed to get "stuck in a rut" and could not work out what he needed to do to put it right. He selected wiggle all - which moves the backbone and the sidechains of the protein randomly - but soon he "stopped wiggle all because the protein seemed to be unfolding instead of folding (Figure 6). 


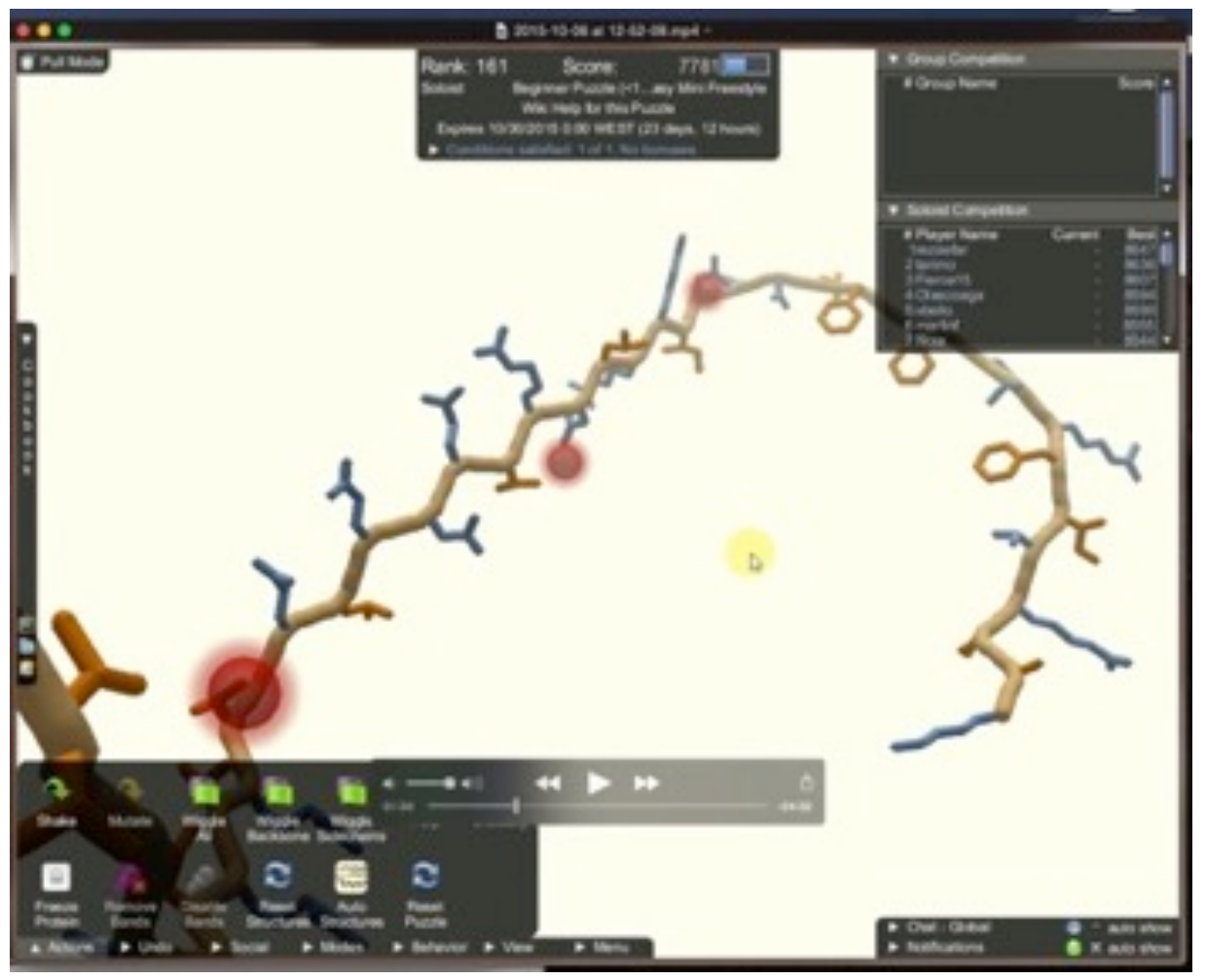

Figure 6. Screenshot showing conflicts (red spots)

IS remarked: "No idea why and when this happens, but it might be because my initial hand-folding wasn't correct so the algorithm is trying to (unsuccessfully) fold the protein but it gets conflicts." Thus he rotated the protein to see the whole chain and observe the conflicts, then he manually solved them, again using random actions and a trial-and error strategy. Throughout the gameplay, IS had been concerned with the change in his score. Since he had not been able to move it up, he decided to try some recipes. He chose a certain recipe that had showed good results in his previously played puzzles, but soon he stopped it because he remembered he should have first unfrozen the whole protein. After correcting this problem manually, from his sparsely populated Cookbook he selected another recipe that had worked on previous puzzles, always keeping an eye at the change in the score. Since the score had not increased for some time, he stopped the recipe and ran it again after rotating the protein. He soon stopped the recipe since he saw no movement in the structure of the protein nor increase in the score. After a short hand-folding that did not increase the score, IS abandoned the game, as he "was unsure how to proceed with folding it." 


\section{Intermediate: Assessing the Change in the Score}

LB started the puzzle with some hand-folding and observation, "on the first day, I designed a structure with 2 sheets and 3 helices. I worked out quickly that it would pull itself apart and I had no time to try and stabilize it." After abandoning her first design, LB decided to make one long helix. As she said "this was a beginner puzzle so I thought that this may be just a small section of a bigger complex, therefore I wouldn't worry about trying to 'bury' the hydrophobic amino acids (AAs)." LB refers here to the Foldit beginner tutorials which teach players that hydrophobic AAs like to be buried away from the water environment and hydrophilic AAs like to be on the outside in the watery environment. LB is still learning by experience and playing evolving team puzzles which recipes to use in the correct sequence. She has learned that some recipes are better at the beginning of the game to keep the protein flexible, while others are only used in the endgame as they lock the protein up. After observing the structure of the protein guided by Foldit color sequence for scoring segments (red=worst, orangey/brown, shades of green=good, bright lemon=best), she decided to run a recipe called QuickFix to select the red worst scoring segments and get rid of them on the end of the protein. QuickFix brought a positive change in score and the red segments turned green. However, she noted that helices were bent and their shape was not ideal. Trying to make a more ideal secondary structure, she ran a recipe that gradually twists a bent helix a few segments at a time then shaking it. However, she forgot that the recipe has a bug and the computer crashed. After restarting the puzzle, she tried several recipes to get the score to increase. Before going to bed she set a recipe called Worm on low behavior to run overnight, "as this recipe often gets the puzzle out of a local minimum." LB explained that this recipe performs a local wiggle sequence by patterns, and if the result is positive, it performs more local wiggle shake moves. As she recalled, "I needed this recipe to work overnight, so I asked the script writing members of Go Science to edit the recipe so that the script would run until I told it to stop. A team member did this for me and now I often use it when a puzzle is expiring during the night." Worm 
did not get the score to move up. Then, LB used another Lua recipe that moves the segments around and shakes them out. Players tell the recipe the number of segments to cut. As a result, the score went back to best before the crash. She also noted that the color of the protein changed more towards the green. Several recipes ran without score gain. "In desperation I was trying any recipe that had gained me points in the past," she recalled. Then, she tried a recipe called Walker, because it had often given good results. She learned this tactic "by watching a Veteran Chat that a 'walking' recipe doesn't stiffen the protein but a 'Walker' recipe does stiffen the protein thereby locking up its potential too early." The "Walker" produced mixed scoring results. Trying to knock the protein out of a local minimum, LB ran another recipe that pulls protein segments in random directions then shakes them out. She noted a score gain. Only eight hours into the puzzle expiry, she sat an overnight recipe which generated only a small score gain, and then she sat again another one, "a very slow recipe that walks along the protein and tweeks the rotomers on the AAs to try and get better angles." She completed the puzzle with the score 8705.973 , which was eight points ahead of the top beginner score of 8698 and 110 points ahead of the expert player.

\section{Expert: Observing and Assessing the Structure of the Protein}

In the beginner's puzzle, BK spent a limited amount of time on monitoring the game, since he only had little information to use and decide whether to run - or stop - a recipe. The main sources of information were the structure of the protein and the recipe output.

As he often does when starting a puzzle, BK hand-folded the protein for a little while, trying to give it "a beautiful and realistically possible look." BK has learned by experience what visual design(s) make a protein beautiful and realistically possible. He noted that the protein "should be aesthetically, "like a dance", with straight and parallel helices and sheets that follow each other in one path with a kind of harmony. Even if a beginner did not know what to do, he would be able to classify bad and good designs based on pure aesthetics, whereby computers would only try to rise the score ${ }^{32}$." Error! Reference source not

found. shows examples of nice designs versus good scores (images sorted by 
score from left to right): a) a nice start game design with a very bad score of 1722 points and dark red spots; b) a bad start game design with a high score of 8576 points: a computer would retain this higher scoring solution to go further on, but a human would prefer the previous design; c) endgame ranked $2^{\text {nd }}$ solution with a score of 9474 points. So (a) was a better candidate than (b).

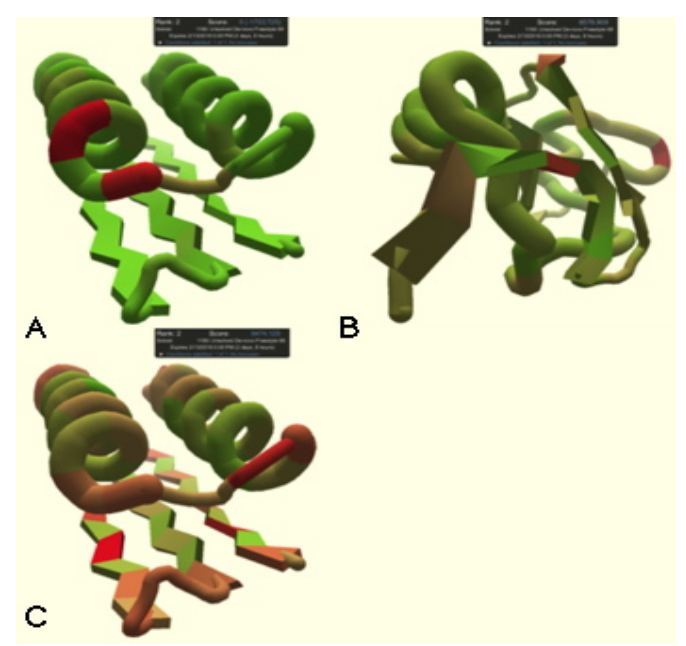

Figure 7. Nice design versus good score.

Soon after, BK ran what he defined "a strong, disturbing recipe able to test different macro structures and keep the best one as a starting point for further "micro" optimizations. It replaces hand-folding on start game." He noted that this recipe could not destroy the minimum scoring structure built with the initial handfolding, and saved him time. BK ran recipes on start game to save time, as he described here: "because of lack of time, I start some recipes very soon (then I have to correct the result manually afterwards). As a puzzle lasts for only about seven days, it's better to run a recipe overnight or during working days than waiting eight hours to come back for further handfold." In the quote above, BK noted the need to correct the outcomes of the recipes he has run. His videorecording showed several instances of repairing mistakes caused by recipes to the structure of the protein. For example, after running a recipe on start game for several hours, he noted red segments in the protein (red color shows poor scoring segments). He ran QuickFix to remove those red dots without destructuring the protein, for this reason he chose the option "never 
wiggle all backbones" in the recipe list of commands, which means not to move the chain of the amino acids, that is, the backbone, around.

During midgame, BK ran QuickFix several times to remove red spots, after observing the outcome of previous recipes on the structure of the protein.

Observation and assessment of the structure of the protein at midgame and in the end of the game also led the player to run recipes. At midgame, after running Quickfix, he observed that a sheet (a flat part of the protein) was lost. He also noted that the protein was stable enough to run a band recipe (bands are tools used to pull parts of a protein together with the use of a wiggle tool that moves the backbone and the sidechains around). By running such a recipe, BK wanted to "change slightly the macro structure without disturbing it too much (e.g. change the distance between two sheets without changing the sheets themselves too much)." As endgame routine, BK ran recipes to refine (tune) the structure without changing the visual design of the protein and optimize the structure of the protein. As he described, performing routines "comes from community experience or tradition basically, and it is not to be contradicted with personal experience." $\mathrm{He}$ stopped playing the puzzle before the end with the score 8596 , which was 51 points below the top beginner score at that time (8647) and 110 points below the final top score 8706 reached by LB. BK found interesting to mention this result as it shows that there is still something random that makes the game attractive: a top player is statistically better, but for each individual puzzle any intermediate player has a chance to gain points.

\section{Findings: Advanced> Unsolved De-Novo FreeStyle 58}

In this narrative, BK described his different strategies to solve this challenging puzzle. He played it both as solo and as evolver within his team Go Science.

Figure 7 summarizes the results of the axial coding analysis related to the most frequent actions he performed in association to "running a recipe." 


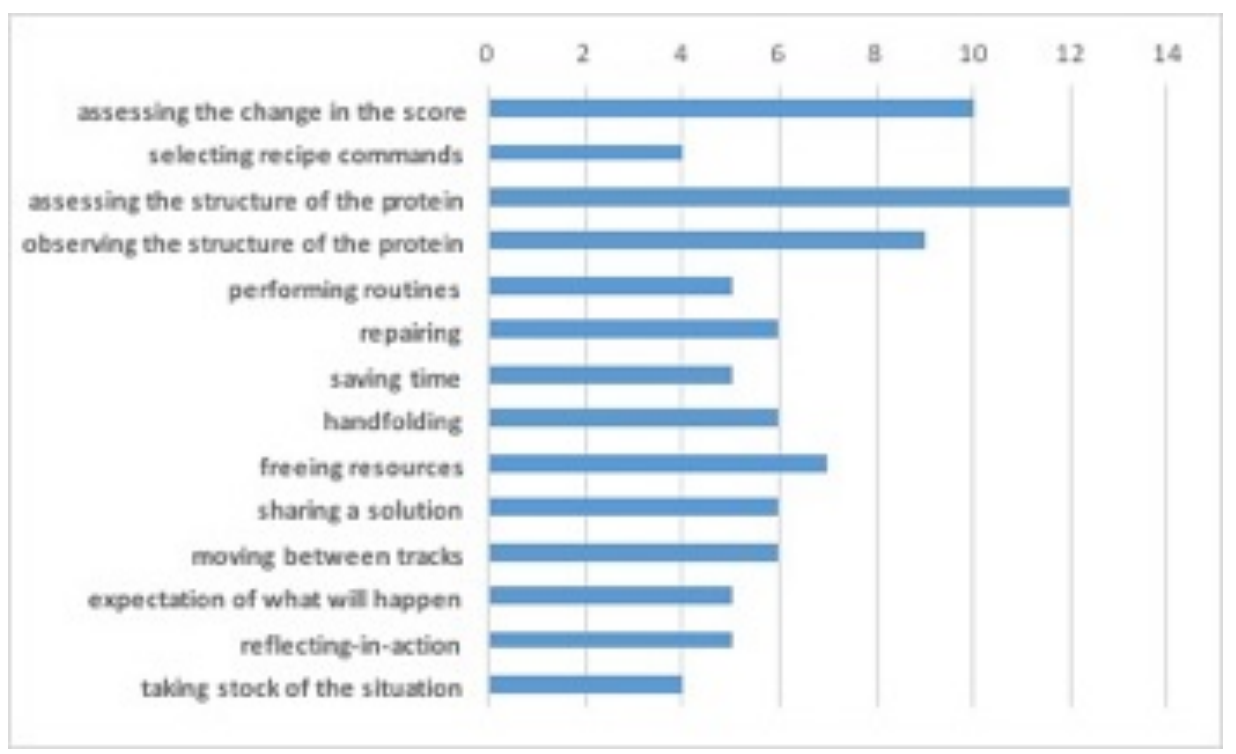

Figure 7. Actions associated to running a recipe in the advanced game

While the list of the observed actions associated to running a recipe includes 34 main concepts, we included in the chart only those that occurred at least four times, because they captured the more important elements of the way in which BK ran recipes. Since the player played this puzzle in a competitive mode (unlike the beginning puzzle, where he was not involved in a competition), assessing and observing the structure of the protein and assessing the change in the score were the most recurrent actions.

Figure 8 shows the occurrences of the external sources of information used when performing the actions shown in Figure 7. The structure of the protein, the score and the recipe output were the three sources used most frequently for deciding to run a recipe. 


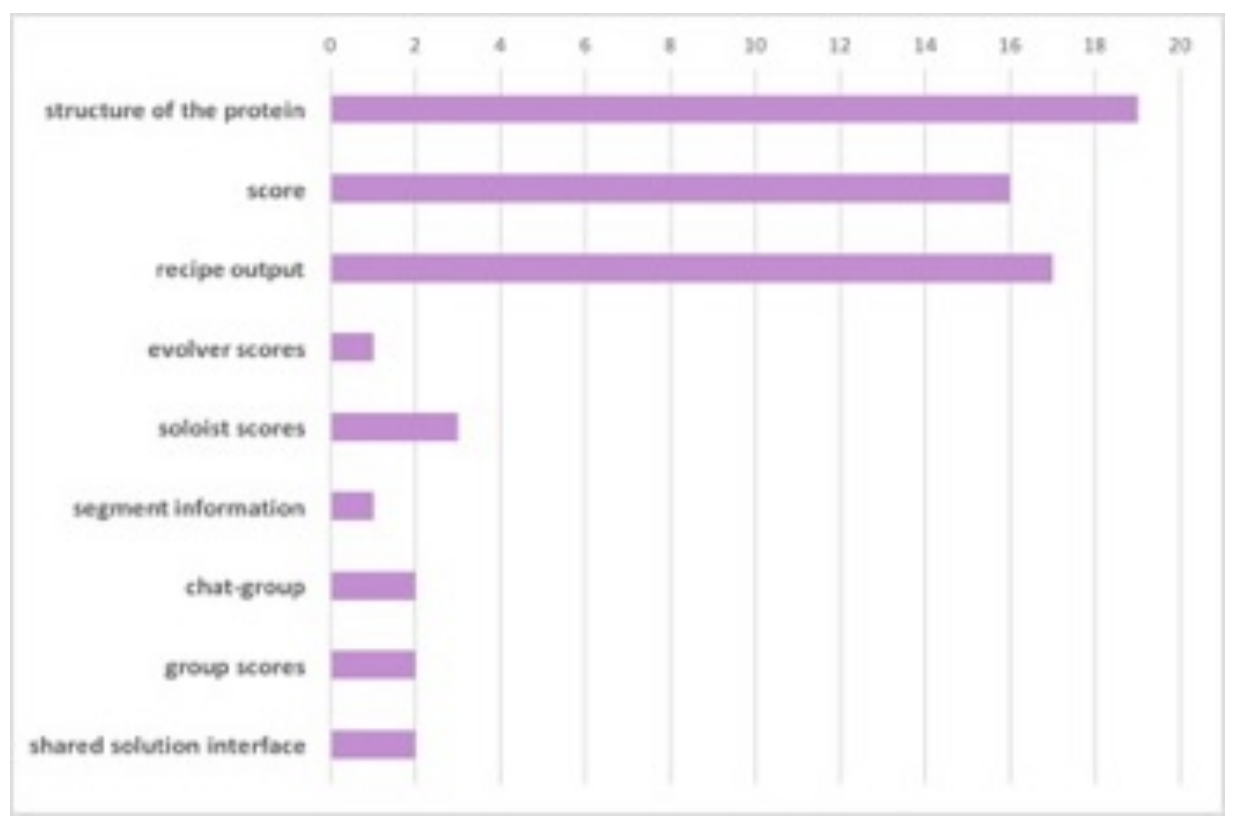

Figure 8. Most frequently used external sources of information

The following narrative describes BK's professional visions through his practices of coding, highlighting and producing material representations.

\section{Seeing and Interacting with Material Representations}

The puzzle opened with some indications on the primary sequence and the possible secondary sequence (which also includes sheets and helices) of the protein. From this flat protein, players are asked to figure out the ideal 3D fold structure. "If we find the ideal structure, the researchers can deduce the function of this protein in nature. This is how Foldit has become popular, when players found a protein structure after 7 days, whereby researchers had not succeeded after ten years," BK said. BK knows that the starting design is essential for further score gains. Therefore, he hand-folded the protein for about 20 minutes at the start of the game. From this basic design on, he worked mainly with recipes till endgame. Additional manual interventions were limited during the seven days of the competition. The start of the game is a phase in which the player mulls over alternatives, as BK describes: "about 20 minutes playing now. No more time left to play and no precise ideas what to do now. So I ran a recipe called Find Starting Point. Now I can leave my computer doing the job during minimum a full day or night." 
During the entire gameplay, BK opened and closed multiple tracks to try new designs. Tracks are a feature that allows multiple game play paths remain independent of each other. They support the players' ability to try multiple approaches to puzzle solving, either from the beginning of a puzzle or branching off somewhere along the way with multiple strategies. In total, he tried three basic designs and for each of them the he tried different strategies at different steps of the game. In each strategy, observing and assessing the structure of the protein and the change in the score were intertwined with selecting and running recipes. For the sake of simplicity, we provide two examples of this intertwinement. BK ran a recipe because he did not have time to manually fold a protein showing a lot of orange outside (all the sidechains of amino acids that fear water are orange and should be inward-facing). The recipe produced good results on score and protein design, so he commented, "after «only» 19 loops of the recipe, the result of B2 (one track) is good either in points (one of the best of my team) and in the protein look. The long curved helix on right front could be separated in two helices separated by loops. I can do it manually later on, after running a "Deep Rebuild Worst" recipe." Our player trusted this recipe because he thought all players use it. The recipe gained some points. Then BK tried a couple of other recipes but was unconvinced and said, "none of these recipes actually selects and bands to the orange hydrophobic sidechains. I'll have to adapt these [recipe] scripts to do that. On my TO DO list!"

At midgame, BK used a track to open the results generated by a recipe on another track. On the former, he ran another recipe to get rid of the red dots in the protein. Although this recipe helped gaining points quickly, he noted that "I should not let it run too long in order not to stiff the protein. By the way, observing the protein. I should make some helices longer, shortening some loops. I stopped the recipe. I manually refined the secondary structure."

Figure 9 zooms in the last four days of the game (our player and his group ranked $2^{\text {nd }}$ at the end of this puzzle, the top score is showed in orange for comparison). At that stage, most players achieved a score above 8000 pts. BK said, "the 
progression is slow, and it is what we invested the first days (with hand and recipes) that will pay now."

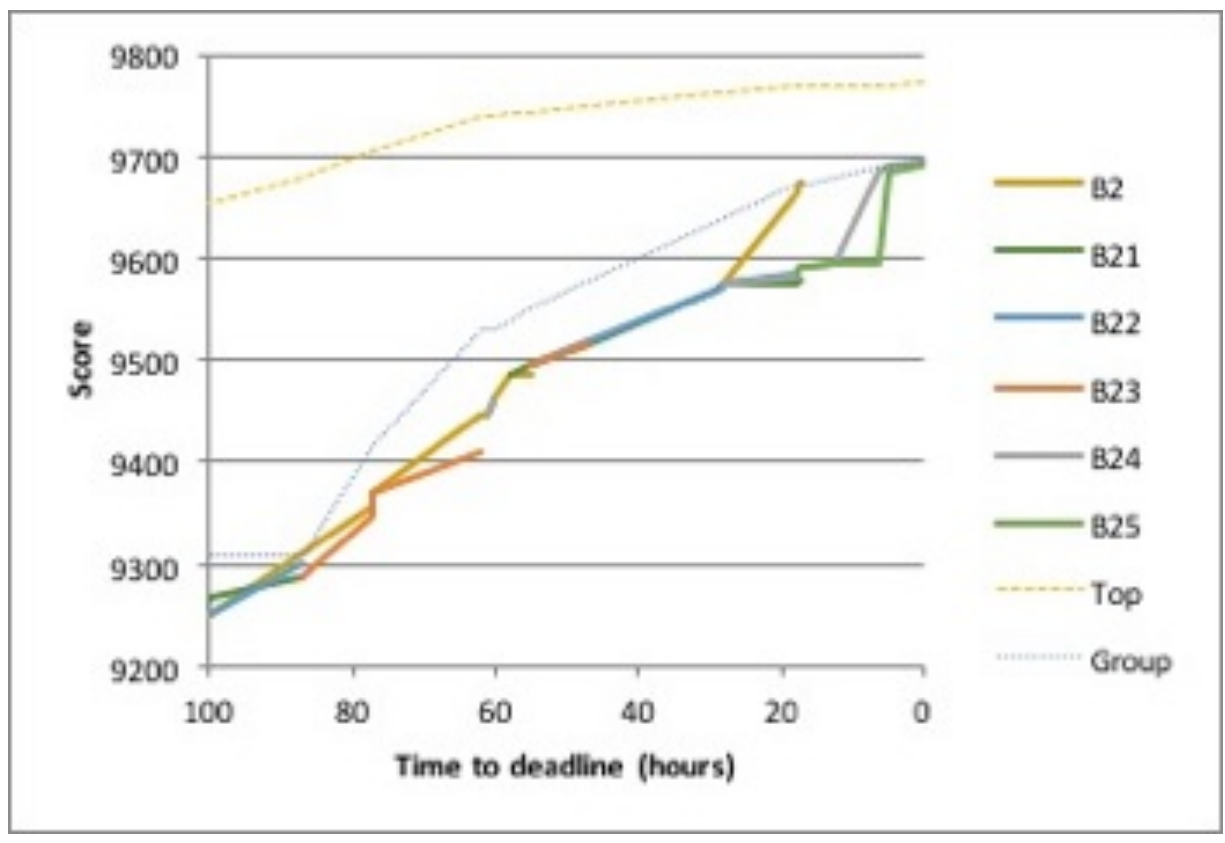

Figure 9. Progression of track scores (Source: Bruno Kestemont)

Figure 9 shows that the player tried up to five different recipes on corresponding tracks from the same node (B2), before selecting the best one as a new node for further progress. BK explained the graph this way, "typically, during the game, I could run recipe 1, recipe 2 and recipe 3 on the same node on three different tracks. If recipe 1 gains more and recipe 2 gains a reasonable amount, I could start from the end of recipe 1 and try again recipe 1 and recipe 2 from there. So the different tries are not missed tries: they help learn how this specific puzzle reacts on recipes, they help to select recipes on further steps".

\section{Discussion and Conclusion}

The main purpose of this study was to investigate players' professional vision and interpret their use of recipes during their gameplay of Foldit in order to add knowledge to debate on whether running recipes is all that is needed to play the game, or whether recipes actually allow skilled Foldit players to strengthen their role of experts rather than becoming appendices of automated gameplay. In order to answer this question, we have first drawn attention to the literature positing that skilled relationships with technologies need to be understood in their 
enactment, instead of "decomposing" skills, tasks, and technologies into entities that do not mutually influence each other. Turning to Goodwin's notion of professional vision, we conceptualized and studied skills as abilities and forms of knowledge being performed through a network of connections-in-action involving humans and technologies.

Thereafter, we looked at Foldit players' practices of seeing and organizing their gameplays. The analysis of what three players (beginner, intermediate, and expert) observed and did visualized the professional vision necessary to use recipes sensibly and effectively. Although professional vision was a useful concept in this study, we should also clarify a main difference between our case and the cases Goodwin examined. Foldit players are not involved in the same focused, collaborative practices used by archeologists for the development of their professional vision. Players play individually, although they can share solutions and consult each other in real-time when playing a puzzle.

Our findings highlight three key abilities:

(a) seeing beauty;

(b) repairing, and

(c) monitoring a large quantity of information to perform actions effectively.

Seeing Beauty

First, a specialized "way of seeing" the structure of the protein is necessary to run recipes successfully. Expert and intermediate players look at the structure of the protein and give salience to specific elements that occur at a given point in time during the game, such as, for example, the shape or position of the backbone, the elements defining the shape of the backbone, the colors of sidechains, the form of helices and the alignment of sheets. Over the years, the expert player has developed a strong ability to observe and assess meaningful events in the structure of protein. This ability is not just the outcome of his individual and cognitive processes, but a situated activity he accomplishes using specific information pointers when playing. The skill of seeing the "beauty" of the protein is a clear example of such situated activity: human spatial awareness, intuition and experience intertwine with material representations to capture beauty and 
harmony. Seeing beauty is a polimorphic action (Collins, de Vries, \& Bijker, 1997) that players develop and master only through discerning what is wrong in a protein, through zooming in and rotating a protein even when scripts are running. Advice and instructions may aid the mastery of this action, but cannot replace experience and intuition. Nor can seeing beauty be decontextualized and inscribed in a recipe. As Figure 7 indicates eloquently, computers are good at calculating good scoring solutions. Recipes can perform what Collins, de Vries, and Bijker call mimeomorphic actions such as wiggling and shaking proteins. These actions can be identified, easily explicated and delegated to recipes, which players perform to have a cost-effective allocation of their time and effort.

\section{Repairing}

Recipes perform actions for a long time to get players closer to their goals. For example, they can fix errors made by other recipes. However, repairing errors is not just a computational action but involves human judgement. Recipes calculate what is wrong, but players need to spot mistakes in the first place and select the right recipe accordingly, depending on the type of problem to be fixed. While recipes can help detect good shapes and allow players to make rapid progress to the solution of puzzles, they are said to do little to fix fundamental flaws which require intelligent intervention, something that is notoriously difficult to do with software (Ponti \& Stankovic, 2015).

\section{Monitoring Large Quantities of Information}

A skilled player can monitor a large quantity of information to make effective decisions, including running recipes. To a beginner, the many sources of information available in and through the game interface seem complex and opaque. The novice is less capable of monitoring this complexity and progress in the game. What appears as a jumble of numbers, text and protein movements to the beginner becomes slowly understandable to the intermediate player and is familiar to the expert. The skill of monitoring this great intake of information entails the ability to make sense of information - for example, the stream of data produced by recipe outputs - and reflect upon the significance for setting goals and taking further actions. A player who has not developed this skill - such as 
our beginner - just engages with a puzzle, moves through it and acts in it, without being able to coordinate the information provided by the many external sources of information and focusing mainly on the score. Interpreting the meaning of the score in Foldit is only apparently intuitive, since the score is a sum of the scores of each segment of the structure of a protein, plus 8000 . Nevertheless, less experienced players can see the score as a more straightforward information than the structure of the protein.

Monitoring large quantities of information from different sources involves the selective use of specific information to perform certain tasks at different stages of the game. The examined accounts show that the expert - and to a less extent the intermediate - know how to manage specific sources of information at different stages. They have learned how to change strategies if they do not meet their goals, use time more efficiently, assess the outcomes of recipes and choose future recipes based on what they had experienced before. Experienced players know how to combine scripts and hand-folding at the right time, perhaps abandoning good scoring solutions when they do not look visually nice. However, experts also run good scoring solutions in parallel because sometimes this is the only way to rank well. Conversely, the beginner relies mostly on random actions, although the expert and the intermediate also perform some random tries. When players face a new challenge, try a new recipe, or have abundant computational resources to work with, use random actions.

To conclude, although recipes embed a number of simple, time-consuming and repetitive manual actions, they cannot replace yet the human skills needed to address the complexity of the game. Seeing beauty, repairing and monitoring a great quantity of environmental information are capabilities that humans learn over time, through training and playing the game intensively. They cannot be mimicked by existing recipes.

While Linderoth's (2010) warned us that in many cases players do not have to learn much to progress in a game because the game can point out many affordances, this study shows that players indeed have to develop a professional vision independent of what the game itself can highlight. This is related to the 
nature of the Foldit game where it is impossible for the game developers to show the affordances, because they are unknown. Players must learn to see the affordances, and develop a professional vision, which means that they have to learn these skills through gaming. Only players that have developed such a professional vision, are able to use recipes to their advance.

An important design implication is that citizen science games like Foldit that aim to identify few talented individuals from a large pool of candidates in the general public and educate them to accomplish megatasks should support beginners' development of professional vision. This can initially be done by highlighting affordances for known solutions and helping players develop an initial sense of beauty, repairing skills and the ability to monitor large quantities of information. This approach will ensure that the initial pool of candidates does not decrease too early. However, later on, in order to help the most talented players develop into experts, such highlighting of affordances should be diminished.

\section{Acknowledgments}

This research was supported through funding from Marianne and Marcus Wallenberg grant no. 2013.0020.

\section{References}

Bonney, R., Shirk, J.L., Phillips, T.B., Wiggins, A., Ballard, H.L., MillerRushing, A.J. \& Parrish, J.K. (2014). Next steps for citizen science. Science, 343 (6178), $1426-1437$

Bravermann, H. (1974). Labor and Monopoly Capital: The Degradation of Work in the Twentieth Century. Monthly Review Press: London \& New Work. Brown, A. (2015). Awkward. In P. Lankoski and S. Björk (Eds.), Game Research Methods - An Overview. ETC Press

Carmeli, A., \& Tishler, A. (2006). An empirical analysis of the relative importance of management team. International Journal of Manpower, 27, 9-36. Charmaz, K. (2006). Constructing grounded theory: A practical guide through qualitative analysis. London: Sage.

Che Pee, N. (2011). Computer games use in an educational system (Unpublished doctoral dissertation). University of Nottingham, Nottingham, England. 
Collins, H. M., de Vries, G. H., \& Bijker, W. E. (1997). Ways of going on: An analysis of skill applied to medical practice. Science, Technology, \& Human Values, 22(3), 267-285.

Cooper, S. (2014). A Framework for Scientific Discovery Through Video Games. New York, NY: Association for Computing Machinery and Morgan \& Claypool. Cooper, S., Khatib, F., Treuille, A., Barbero, J., Lee, J., Beenen, M., ... Foldit players (2010). Predicting protein structures with a multiplayer online game. Nature, 466, 756-760. doi:10.1038/nature09304

Cooper, S. Khatib, F., Makedon, I., Lu, H., Barbero, J., Baker, D., ...Foldit Players. (2011). Analysis of social gameplay macros in the Foldit Cookbook. In FDG'11, Proceedings of the 6th International Conference on Foundations of Digital Games (pp. 9-14). ACM, New York, NY.

De Paoli, S. (2013). Automatic-play and player deskilling in MMORPGs. Game Studies: The International Journal of Computer Game Research, 13(1). Retrieved March 23, 2015 from http://gamestudies.org/1301/articles/depaoli_automatic_play

Good, B.M., \& Su, A.I. (2013). Crowdsourcing for bioinformatics.

Bioinformatics, 29(16), 1925-1933. doi: 10.1093/bioinformatics/btt333

Goodwin, C. (1994). Professional vision. American Anthropologist, 96(3), 606633.

Gherardi, S. (2012). How to conduct a practice-based study. Cheltenham, UK: Edward Elgar Publishing.

Hand, E. (2010). Citizen science: People power. Nature, 466, 685-687. doi:10.1038/466685a

Khatib, F., Cooper, S., Tyka, M. D., Kefan, X., Makedon, I., Popovic, Z., ... Foldit Players. (2011). Algorithm discovery by protein folding game players. PNAS, 108(47), 18949-18953, doi: 10.1073/pnas.1115898108 Linderoth, J. (2010). Why gamers don't learn more: An ecological approach to games as learning environments. In Proceeding of DiGRA Nordic (Stockholm, 2010). 
Poteete, A. R., Janssen, M. A., \& Ostrom, E. (2010). Working Together:

Collective Action, the Commons, and Multiple Methods in Practice. Princeton, New Jersey: Princeton University Press.

Prestopnik, N., \& Crowston, K. (2012). Purposeful gaming \& sociocomputational systems: A citizen science design case. In GROUP'12 Proceedings of the $17^{\text {th }}$ ACM International Conference on Supporting Group Work (pp. 75-84), October 27-31, 2012, Sanibel Island, Florida. doi>10.1145/2389176.2389188. Reeves, S, Brown B. \& Laurier, E. (2009). Experts at play: understanding skilled expertise. Games and Culture, 4(3), 205-227.

Rouncefield, M. Slack, R., \& Hartswood, M. (2011). Technology. In M.

Rouncefield \& P. Tolmie (Eds.), Ethnomethodology at Work (pp. 191-210), Ashgate Publishing Group: Farnham, UK.

Schell, J. (2008). The Art of Game Design: A Book of Lenses. Burlington, MA: Morgan Kaufmann Publishers.

Scott, K.S. (2004). Relating categories in grounded theory analysis: Using a conditional relationship guide and reflective coding matrix. The Qualitative Report, 9(1), 113-126.

Tedder, V. (2016). Digital stories and handmade skills: explorations in how digital methods can be used to study transmission of skills. In H. Snee, C. Hine, Y. Morey, S. Roberts \& H. Watson (Eds.), Digital methods for Social Science (pp. 157-170). Palgrave Macmillan: Houndmills, UK.

von Ahn, L. (2005). Human computation (Unpublished doctoral dissertation). Carnegie Mellon University, Pittsburgh, PA. 
${ }^{1}$ See the pages "What's the point? Can't this just be automated using recipes?" and "Hand-folding vs. scripts: The Dishwasher Analogy" in Wikia Foldit.

${ }^{3}$ For illustrations of the aesthetics of the 3D structure of known proteins, see Fox, N. K, Brenner, S. E, \& Chandonia, J. M. (2014). SCOPe: Structural Classification of Proteins - extended, integrating SCOP and ASTRAL data and classification of new structures. Nucleic Acids Research 42: 304-309. Database on: http://scop.berkeley.edu 